\title{
Investigación-acción como metodología para el diseño de un serious game
}

\section{Action research as a game design methodology for a serious game}

\author{
Ruth S. Contreras-Espinosa \\ Universitat de Vic-Universitat Central de Catalunya, Uvic-UCC (España) \\ José Luis Eguia-Gómez \\ Lluis Solano Albajes \\ Universitat Politècnica de Catalunya, UPC (España)
}

\section{Resumen}

Se ha producido un importante desarrollo en la investigación relacionada con los juegos educativos y, en especial, con los serious games y el aprendizaje basado en juegos digitales durante la última década. De particular interés son las posibilidades que proporcionan este tipo de juegos para involucrar a los estudiantes en la experiencia de aprendizaje. Los avances recientes incluyen desde desarrollos teóricos hasta la creación de juegos digitales para una amplia gama de planes de estudios o la aparición de entornos de aprendizaje basados en juegos tanto para la educación formal como para la formación de personas. Pero todavía hacen falta principios y propuestas para la investigación y el diseño de serious games, experimentos y metodologías basadas en el diseño de juegos. En este artículo presentamos una experiencia llevada a cabo por un grupo multidisciplinario de investigadores en colaboración con profesores de primaria de la ciudad de Barcelona, España, para desarrollar un juego digital que permite trabajar competencias de educación orientadas a estudiantes de primaria. Hemos diseñado un juego digital utilizando un proceso de cocreación con base en la metodología investigaciónacción. El juego llamado Ferran Alsina se creó como un facilitador de una nueva metodología de enseñanza y se centra en trabajar con competencias básicas, metodológicas y específicas para niños de primaria, con edades entre los 8 y 11 años de edad.

Palabras clave: juego educativo; metodología; investigación-acción; educación primaria; participación del profesor.

\begin{abstract}
There has been significant development in educational games, especially with serious games and digital game-based learning in the past decade. Of particular interest is the potential that serious games have for engaging learning experiences. Recent advances include theoretical developments, the creation of game-based learning environments for a broad range of curricula, and the emergence of technically advanced game-based learning environments for both education and training. But still we need more principles of design-
\end{abstract}


based research and design-experiment methodologies. In this paper we present an experience of co-creation carried out by a multidisciplinary group of researchers in collaboration with primary teachers from Barcelona (Spain) to develop a digital game to work on primary education-oriented competencies. We designed a co-creation design game based on action research methodology. The serious game called Ferran Alsina was created as a facilitator for a new teaching methodology and is focused on to work with basic, methodological and specific skills for elementary students, 8-11 year-old children.

Keywords: educational game; methodology; action research; primary education; teacher participation.

Desde hace años, las instituciones de educación se han visto inmersas en un proceso de innovación que incluye la introducción de juegos digitales en las aulas, pero además, una reforma curricular que ha modificado sus programas de estudios. Es en este panorama donde los serious games tienen la posibilidad de mostrar todo su potencial. El amplio interés por este tipo de juegos, así como por el aprendizaje basado en juegos digitales, incluye desde desarrollos teóricos (Gee, 2007; Gibson, Aldrich y Prensky, 2007), hasta la creación de diversos entornos pensados en una amplia gama de planes de estudios (Kebritchi, Hirumi y Bai, 2010). La investigación ha madurado y puede decirse que los estudios empíricos ya han demostrado que se pueden lograr resultados significativos en el aprendizaje gracias a la interacción y a actividades que se llevan a cabo en diversos juegos digitales centrados en una gran variedad de temas (Johnson, 2010). Sin embargo, todavía necesitamos más principios y propuestas para la investigación y el diseño de experimentos y metodologías basadas en el diseño de juegos (Arnab et al., 2015; Perrotta et al., 2013). Por ello, hemos realizado una experiencia de cocreación con el propósito de contribuir y ofrecer una propuesta participativa para el diseño de serious games.

La propuesta que presentamos está basada en la metodología investigaciónacción. Gracias a la acción y reflexión, la teoría y la práctica y a la participación de personas que buscan un mismo fin, el resultado ha sido una solución útil, para el diseño de un juego. El serious game diseñado gracias a esta experiencia se orientó a alumnos de quinto y sexto de primaria y se incluyeron en su contenido competencias del currículo de educación de Cataluña. El equipo de investigación contó con la participación de veinticinco profesores de la zona de Barcelona. Principalmente fueron ellos, como usuarios, quienes influyeron en el proceso de cocreación al participar en tareas como la generación y evaluación de ideas, la implementación de competencias dentro de la jugabilidad y la narrativa del juego, o el desarrollo del prototipo. La experiencia dependió de los participantes (Kohler, Fueller, Stieger y Matzler, 2011), y se produjo durante el proceso de diseño en referencia a las actividades conjuntas de desarrollo. 
Se plantearon los siguientes objetivos:

- Desarrollar un proceso de cocreación en el que los participantes contaran con un papel activo en la innovación. Se consideraron usuarios a los profesores de primaria, porque son los responsables de que los juegos lleguen a los alumnos y aulas.

- Seleccionar las competencias del currículo del departamento de enseñanza de Cataluña que se introducirían en el juego.

- Adaptar el serious game a las necesidades pedagógicas específicas del ciclo superior de primaria utilizando competencias. Se tuvo en cuenta una jugabilidad atractiva para los alumnos.

- Definir la dinámica de trabajo para el proceso de cocreación.

El artículo se encuentra estructurado en cinco partes. Explicamos el fundamento teórico en el cual se sustenta la propuesta. Describimos la metodología utilizada así como las diferentes fases y los ciclos inmersos en la metodología investigaciónacción. Explicamos el juego resultado de la experiencia, así como las competencias incluidas en el serious game. En el antepenúltimo apartado discutimos los resultados obtenidos, las limitaciones del proyecto y el trabajo futuro. Finalmente, presentamos las conclusiones a las que hemos llegado.

\section{BASE TEÓRICA}

\section{Serious Games}

La definición más conocida nos indica que los "serious games" tienen una finalidad educativa explícita y están cuidadosamente pensados para no ser destinados a la diversión principalmente (Abt, 1970). Se habla entonces de un uso general de juegos digitales y tecnologías con fines más allá del entretenimiento (Sawyer, 2007). Según Zyda (2005), son vistos como un ejercicio mental jugado en un ordenador, de acuerdo con unas normas específicas basadas en el entretenimiento e incorporadas en la formación empresarial, la educación, la salud, la política pública u objetivos estratégicos de comunicación.

Abt (1970) destaca que deben considerarse cuatro componentes estructurales en un juego: objetivos, reglas, retos e interacción. El uso de objetivos, reglas y retos en un juego determinará el orden, los derechos y las responsabilidades de los jugadores. Además, permitirá al jugador enfrentarse a problemas para los cuales tendrá que buscar soluciones.

Con respecto a la interacción, en un juego, este componente surge de la propia mecánica y dinámica, y da lugar a las vivencias de cada jugador. Estas experiencias emergerán como resultado de la valoración que tendrá el usuario con el juego, que 
es una actividad libre que se realiza sin una obligación externa. Dicha actividad incentivará al alumno a experimentar, a probar múltiples soluciones, a descubrir la información y nuevos conocimientos sin temor a equivocarse. Los serious games, por tanto, están alineados con la filosofía del constructivismo, ya que permiten al jugador construir su propia comprensión del mundo (Michael y Chen, 2006). Como ya se ha comprobado en diversos estudios (Arnab et al., 2015; Perrotta et al., 2013), gracias a un juego de estas características un estudiante puede desarrollar actitudes positivas hacia los contenidos presentes y puede mostrar un mayor interés sobre estos al ser tratados en otras actividades fuera del juego. Pero además, permite trabajar con competencias específicas (Eguia-Gómez, Contreras-Espinosa y Albajes, 2014), y actualmente, cualquier propuesta de acción educativa debería enmarcarse en este modelo que rige el diseño de buena parte de los sistemas educativos.

\section{Cocreación y diseño}

Algunos autores defienden que la cocreación está presente en todos aquellos aspectos colectivos de algunos proyectos (Durugbo y Pawar, 2014), y se considera el resultado final de diferentes acciones sobre un mismo producto u obra que tiene el fin de innovar. Según Banks y Potts (2010), la cocreación fomenta la generación de valores, tanto en un aspecto cultural como en un aspecto económico. Podríamos añadir que no hablamos de un concepto que representa a un selecto "focus group" (Banks y Humphreys, 2008), sino a una forma de producción.

Nambisan y Nambisan (2008) muestran un estudio donde las experiencias de interacción de los usuarios, en el marco de foros y productos en línea, proporcionan un apoyo empírico para afirmar que son ellos los que aportan valor a la cocreación y pueden ser una fuente de inspiración. Incluso proponen un marco analítico y sugieren que en los sistemas de cocreación tienen que considerarse cuatro dimensiones: la pragmática, la sociabilidad, la hedónica y la usabilidad. El objetivo de estos puntos no es otro que atender a las necesidades de los participantes. El primer aspecto se refiere a la experiencia del usuario en la realización de objetivos relacionados con el producto en sí, aquel que le permite estar informado e inspire su creatividad. Los aspectos sociales, por su parte, son las relaciones subyacentes de estas interacciones y forman el componente de la sociabilidad. El siguiente punto se relaciona con la interacción dada en los espacios virtuales, que puede ser mentalmente estimulante, con lo que se hace referencia al componente hedónico. Por último, la dimensión llamada usabilidad se define por observar la calidad de las interacciones del ser humano con la máquina. Sobre la base de estos componentes que sugieren un conjunto de estrategias de aplicación para espacios en línea, se construyó un marco básico para iniciar la cocreación, pero se han adaptado a las necesidades particulares de nuestro caso. 


\section{Competencias}

Las propuestas más importantes que impulsan la educación por competencias surgen en Europa: El proyecto Tuning, amparado por la Unión Europea y el proyecto DeSeCo (2005), que promueve la Organización para la Cooperación y el Desarrollo Económicos. Según el proyecto DeSeCo (2005), una competencia es la capacidad de responder a demandas complejas y llevar a cabo tareas diversas de forma adecuada. Supone una combinación de habilidades prácticas, conocimientos, motivación, valores, actitudes, emociones y otros componentes sociales y de comportamiento que se movilizan conjuntamente para lograr una acción eficaz. Esta combinación de conocimientos, capacidades y actitudes adecuadas, que se enmarca en un proyecto de desarrollo personal y colectivo, puede aprovecharse en el desarrollo de un juego digital para trabajar diversos aspectos del currículo por estas razones: Las referencias a la enseñanza por competencias se han ido instalando en las instituciones europeas en estrecha relación con los ejes de renovación de la escuela, unos ejes en los que diversas áreas están trabajando desde hace tiempo (Perrenoud, 2012, p. 2). Una competencia es más que conocimientos y habilidades. Si vemos a las competencias como capacidades para afrontar demandas complejas en un contexto particular, y como resultado de la integración, movilización y adecuación de capacidades, conocimientos o actitudes utilizadas eficazmente en situaciones reales, un juego serio puede ser el mejor medio para ello. Una competencia incluye un saber hacer y un querer hacer en situaciones concretas en función de un objetivo. En este escenario, cobran relevancia las palabras de Barnett (2001, p. 32) cuando resalta: "el cambio consiste, esencialmente, en pasar del conocimiento como contemplación al conocimiento como acción".

\section{Investigación-acción (IA)}

El término "Investigación-acción" se asocia con Kurt Lewin y con una metodología cíclica, dinámica y de colaboración. Kemmis y McTaggart (1988) la describen como una investigación reflexiva colectiva emprendida por participantes en situaciones sociales con el fin de mejorar la racionalidad de sus propias prácticas sociales o educativas, así como la comprensión de situaciones en las que estas prácticas se llevan a cabo. Su naturaleza colaborativa desarrolla ideas prácticas para ayudar a la búsqueda de propósitos humanos que valgan la pena estudiar (Hine, 2013). En concreto, el proceso participativo busca principalmente reunir a la acción y la reflexión, la teoría y la práctica, con la participación de los demás, en la búsqueda de soluciones prácticas a problemas. En general, la Investigación-acción permite desarrollar un enfoque sistemático hacia sus propias prácticas orientado a lograr un cambio positivo (Holter y Frabutt, 2012). Ha sido además descrita como un proceso adecuado para desarrollar principios de diseño para un sistema (Lindgren, 
Henfridsson, y Schultze, 2004) y, dado que nuestro propósito es crear las bases de un serious game, se ha tenido en cuenta el uso de esta metodología.

\section{LA EXPERIENCIA}

El diseño de un juego es una tarea compleja, por lo que para idear un sistema que permita aunar experiencias eficaces y atractivas para el aprendizaje, se pensó en un proceso de cocreación en el marco de la investigación-acción, en adelante IA. El diseño se desarrolló a lo largo de doce meses a partir de septiembre de 2012. La experiencia, en la que participaron veinticinco profesores de quinto y sexto de primaria del área metropolitana de Barcelona, duró seis meses. Mediante grupos de discusión pudieron obtenerse recomendaciones y experiencias utilizando juegos en las aulas. El tiempo asignado para cada grupo fue de dos horas. El grupo de profesores contaba con una edad de entre 24 y 52 años. Se invitó a los participantes a sumergirse en el contexto del problema, a participar en los retos que el juego podía plantear, además de aportar conceptos e intercambiar ideas.

\section{Ciclo 1}

Se realizó en cinco fases:

- Diagnóstico. Con la intención de identificar experiencias a nivel tecnológico, pedagógico y creativo, en la fase I se partió de una revisión bibliográfica y del análisis de juegos existentes en el mercado.

- Planificación de la acción. En la fase II, se planificó el proceso de cocreación y más tarde se afinó una propuesta teniendo en cuenta los principios diseñados por Nambisan y Nambisan (2008). Los principios se adaptaron a las necesidades particulares de nuestro caso:

A. Pragmatismo: Desarrollar elementos interactivos para situar a los participantes e inspirar su creatividad.

B. Sociabilidad: Fomentar la colaboración.

C. Hedonismo: Proporcionar tareas desafiantes.

D. Usabilidad: Simplificar la experiencia con una estructura clara.

Más tarde, se planificaron los grupos de discusión.

- Acción. En la fase III y, para iniciar el proceso de cocreación, la idea se discutió con los profesores. Con el objetivo de fomentar la participación, la experiencia es fundamental, al igual que el estado mental de los usuarios (Nambisan y Nambisan, 2008). Mediante grupos de discusión se pudo hablar de recomendaciones y 
experiencias, haciendo hincapié en el poder disuasorio de los videojuegos como mundos virtuales. Las discusiones de grupo se integraron para explorar las necesidades de los alumnos, incorporar competencias en el juego y examinar oportunidades. Y tal y como se hace en el diseño de otros productos interactivos, como las aplicaciones, se adaptó el material pedagógico y audiovisual (Ibáñez, Jordano y Vermeulen, 2016). Se concretó más tarde la temática del juego y el personaje. Se pidió a los profesores que expresaran sus ideas y experiencias para participar en el diseño del juego. Los participantes fueron capaces de valorar, comentar y juzgar ideas. La tabla 1 enumera cada uno de los principios.

Tabla 1. Componentes de experiencia

\begin{tabular}{ll}
\multicolumn{1}{c}{$\begin{array}{c}\text { Componente de } \\
\text { experiencia }\end{array}$} & \multicolumn{1}{c}{ Características } \\
Pragmatismo & $\begin{array}{l}\text { Los participantes reciben información preliminar dirigida a } \\
\text { impulsar y preparar el escenario para las siguientes actividades. } \\
\text { Se enfrentan con material para estimular la creatividad: } \\
\text { imágenes y vídeos. }\end{array}$ \\
\hline Sociabilidad & $\begin{array}{l}\text { Se fomenta la colaboración limando las expectativas de los } \\
\text { participantes. Para nutrir los debates y la colaboración, la } \\
\text { discusión comienza cuando los usuarios se sienten preparados. }\end{array}$ \\
\hline Hedonismo & $\begin{array}{l}\text { El participar en un proceso de cocreación se percibe como un } \\
\text { desafío estimulante para los participantes. }\end{array}$ \\
\hline Usabilidad & $\begin{array}{l}\text { Los participantes experimentan el proceso de cocreación. Por } \\
\text { lo tanto, expresan opiniones basándose en una propuesta } \\
\text { prediseñada por el grupo de investigación. }\end{array}$ \\
\hline
\end{tabular}

- Evaluación. Esta fase se centró en los resultados obtenidos, que permitieron comprender los requisitos necesarios para que el juego fuera útil e instructivo. Como Marton (1981) señala, los propios sujetos del estudio son quienes mejor pueden describir las concepciones subjetivas del mundo circundante.

- Especificaciones. El equipo de investigación registró las fases y tuvo en cuenta los puntos desarrollados en la fase III. Basándonos en ellos, se realizó un informe en la fase IV.

Como resultado, el primer ciclo mejoró nuestro conocimiento sobre la puesta en marcha de un proceso de cocreación para el diseño de un serious game, e hizo plantearnos nuevos principios para llegar a la conclusión de que es necesaria una clara comprensión de los sistemas lúdicos y de la tecnología que quiere ponerse en práctica. El marco teórico nos orientó y proporcionó una valiosa comprensión inicial sobre cuestiones generales. Concluimos que necesitábamos una visión más profunda 
y más rica en los detalles con el fin de mejorar la experiencia. Lo más relevante es que pudimos observar que el proceso de cocreación nos obliga a prestar una atención individualizada a los participantes. Por tanto, en la cocreación se ha puesto mayor énfasis en la dimensión de la sociabilidad descrita en los principios de Nambisan y Nambisan (2008).

\section{Ciclo 2}

A continuación se describen las cinco fases:

- Diagnóstico. Con la intención de preparar el diseño del juego, se identificaron los principios de diseño y se observaron tendencias a nivel tecnológico, pedagógico y creativo. En la fase I, se unieron los resultados de la revisión bibliográfica, el análisis de juegos existentes en el mercado y los resultados de los grupos de discusión. Se tuvieron en consideración los componentes estructurales en un serious game mencionados por Abt (1970).

- Planificación de la acción. Se definieron los requisitos necesarios para la creación de un juego útil e instructivo. El equipo inició un documento de diseño que indicaba las reglas del juego, las mecánicas, los personajes, la jugabilidad y las competencias inmersas en el mismo. Estas últimas fueron seleccionadas por los profesores. Se decidió, además, la programación del juego para garantizar la interoperabilidad de la comunidad a la cual va dirigido.

- Acción. Se elaboró una propuesta acorde con las competencias y los procesos de cocreación llevados a cabo en el ciclo anterior. Se diseñó el prototipo para poder realizar pruebas en una fase posterior.

- Evaluación. Se realizaron pruebas de usabilidad y se recopilaron los resultados obtenidos.

- Especificaciones. Se registraron especificaciones, fases y ciclos.

El resultado de este ciclo nos permitió identificar los principios de diseño que enriquecen la propuesta de juego. Lo más relevante fue el desarrollo del prototipo teniendo en cuenta los resultados de los grupos de discusión, los resultados de la revisión bibliográfica y el análisis de los productos existentes en el mercado. Todo esto nos llevó a definir los requisitos necesarios incluyendo la tecnología y el análisis de los dispositivos utilizados habitualmente por los alumnos de primaria en las aulas de Barcelona. Después del desarrollo del documento de diseño, se llevó a cabo el diseño del prototipo, estableciendo objetivos, reglas, retos e interacción y competencias. Finalmente, se reunieron los resultados del estudio de usabilidad. En la tabla 2 se resumen las fases realizadas en dos ciclos. 
Tabla 2. Fases y ciclos del proyecto

\section{Ciclo 1}

\section{Fase I. Diagnóstico}

- Identificación de las posibilidades y alcances de la IA.

- Revisión de literatura asociada. La búsqueda se restringió a artículos relacionados con las palabras clave: serious games, competences, action research y co-creation.

- Análisis de productos existentes en el mercado, a nivel tecnológico, pedagógico y creativo.

\section{Fase II. Planificación de la acción}

- Planificación del proceso de cocreación.

- Planificación de los grupos de discusión virtuales semiestructurados con profesores que utilizan juegos digitales en las aulas.
- Desarrollo de una propuesta teniendo en cuenta los resultados de los grupos de discusión, los resultados de la revisión bibliográfica y los productos existentes en el mercado.

- Definición de los requisitos necesarios para un juego serio útil e instructivo.

- Decisión sobre la tecnología a utilizar con el fin de garantizar la interoperabilidad.

- Analisis de los dispositivos utilizados habitualmente por alumnos de primaria en aulas catalanas.

- Desarrollo del documento de diseño para el juego.

\section{Fase III. Acción}

- Desarrollo de tres grupos de discusión semiestructurados.

- Población de veinticinco profesores.

- Diseño del prototipo, los objetivos, las reglas, los retos y la interacción, así como competencias presentes en el juego.

\section{Fase IV. Evaluación}

\begin{tabular}{l|l} 
- Resultados. & - Pruebas de usabilidad.
\end{tabular}

- Análisis de los resultados obtenidos en los - Resultados y análisis de las pruebas de grupos de discusión. usabilidad.

\section{Fase $V$. Especificaciones}

- Registro de las fases y ciclo 1.

- Registro de las fases y ciclo 2.

Fuente: Adaptada de Eguia-Gómez, Contreras-Espinosa y Albajes (2014).

La IA nos ha permitido aunar esfuerzos para desarrollar ideas prácticas en la búsqueda de un propósito común y reunir acción y reflexión, teoría y práctica con todos los interesados en el uso de un serious game. Paralelamente y como se observó en la literatura, ha sido un proceso adecuado para desarrollar principios de diseño para la creación de las bases de un serious game. 


\section{EL RESULTADO DE LA EXPERIENCIA}

Como resultado del proceso de cocreación, presentamos el juego destinado a niños de 8 a 11 años. El argumento tiene como eje central dar a conocer a un personaje histórico: Ferran Alsina. Fue el técnico de la industria textil y economista catalán a quien se le atribuye el diseño de la fábrica localizada en la colonia Güell. El jugador conocerá el proceso de industrialización en Cataluña. Las acciones que debe realizar son intuitivas y una narración le proporcionará instrucciones detalladas sobre lo que tiene que hacer. Recibirá ayuda constante mediante texto e imágenes que muestran lo que está sucediendo en la partida que, además, muestra las posibilidades para superar dificultades. El juego sigue la estructura de juegos de plataformas que contribuyen al desarrollo psicomotor, la orientación espacial, la dirección y focalización de la atención, así como a la rapidez de respuesta. La estructura incluye 14 niveles con actividades que conforman una narración lineal. En la tabla 3 se muestran los niveles. El juego está diseñado para utilizarlo en plataforma web teniendo en cuenta que los dispositivos más utilizados en las aulas de Barcelona son los ordenadores.

Tabla 3. Niveles del Prototipo

\begin{tabular}{|l|l|}
\hline \multicolumn{1}{|c|}{ Nivel } & \multicolumn{1}{c|}{ Nombre } \\
\hline 1 & El Futuro. Año 3550. \\
\hline 2 & El Vapor Vell. Año 1889. \\
\hline 3 & En Barcelona. Año 1889. \\
\hline 4 & Viaje en Globo por Cataluña. Año 1890. \\
\hline 5 & La colonia Güell. Año 1912. \\
\hline 6 & Viaje en tren. Año 1912. \\
\hline 7 & La mina. Año 1912. \\
\hline 8 & La mina. Año 1912. \\
\hline 9 & El regreso en tren. Año 1912. \\
\hline 10 & Visita a una nueva fábrica. Año 1912. \\
\hline 11 & El puerto de Barcelona. Año 1811. \\
\hline 12 & Alrededor de Barcelona. Año 1912. \\
\hline 13 & Viaje por Cataluña. Año 1810. \\
\hline 14 & De regreso a Barcelona. Año 1809. \\
\hline
\end{tabular}

Nota: Tomada de Eguia-Gómez, Contreras-Espinosa y Albajes (2014). 


\section{Niveles}

Los jugadores podrán seguir la historia desde el primer nivel o adentrarse en un nivel concreto. Todo empieza cuando el jugador accede al juego y observa una introducción en vídeo que le servirá para situarle en la historia. Una vez ésta finaliza, el usuario podrá empezar a superar los primeros niveles del juego. Los tres primeros niveles explican la dinámica. Se busca simplificar la experiencia del jugador mostrando una estructura de navegación fácil e intuitiva, figura 1.

Figura 1. Interfaz gráfica

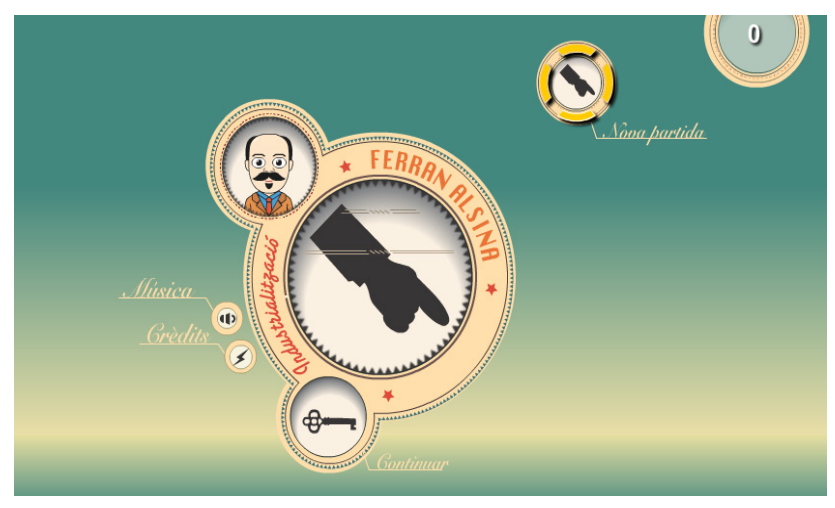

En "El futuro, año 3550", el avatar accederá al juego y al menú desde el cual es posible retroceder a niveles anteriores con el fin de mejorar su puntuación. Durante la partida, el jugador es conducido por un niño llamado Lluc Mercuri. El niño se encuentra escoltado por su robot Yiru. Ambos son personajes del futuro y hacen una regresión en el tiempo para llegar a comienzos del siglo XIX. Una vez situados en el primer nivel, el jugador se sorprenderá observando las energías renovables y comparando las encontradas en el año 3550 con las que se utilizaban en 1889. El segundo nivel, centrado en el siglo XIX, permitirá al avatar descubrir las características de la fábrica "Vapor Vell", figura 2. 
Figura 2. Nivel 2, "Vapor Vell” año 1889

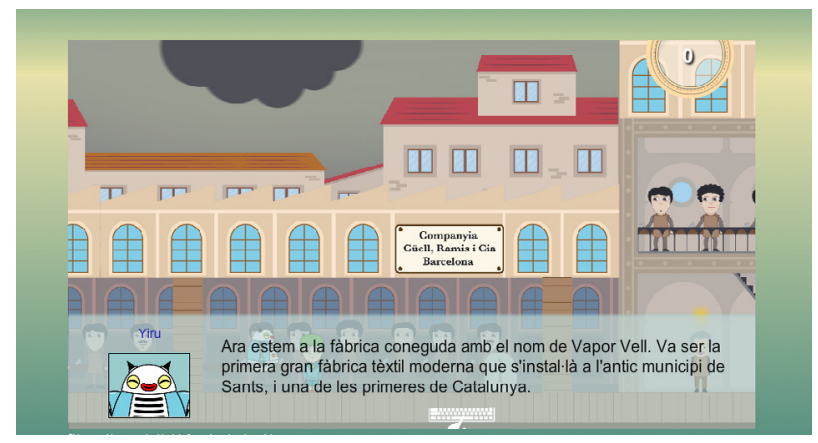

En el tercer nivel, el avatar conocerá las preocupaciones de los obreros por la mecanización de las fábricas. Lluc conocerá la casa de Alsina, y descubrirán juntos lo que es un regulador de Watt. En el siguiente nivel, denominado "Viaje en globo por Cataluña - Año 1890”, el avatar emprenderá un viaje para conocer el impacto de la industrialización en otras ciudades y verá las industrias mineras, de sal y de carbón. En el nivel 5, visitará posteriormente el año 1912, donde conocerá la Colonia Güell diseñada por el arquitecto Antoni Gaudí. Deberá conseguir carbón para mantener caliente a la colonia, con lo que emprenderá un nuevo reto y viajará en tren a un nivel posterior, al nivel 6. En los niveles siete (figura 3) y ocho, el jugador regresará a las minas y descubrirá la actividad que ocurre en ellas, pero también podrá ver las dificultades a las que se enfrenta la industria minera. Más tarde regresará a la ciudad de Barcelona en el nivel nueve. Ya en el nivel diez, el avatar se arriesgará a visitar una nueva fábrica, pero tendrá que evitar los peligros que comporta la instalación de nuevas máquinas. En el nivel once, el año 1811, el avatar visitará el puerto de Barcelona, donde conocerá al Dr. Salvà, un destacado investigador del siglo XVIII, que fue el primer científico del estado español que combatió la viruela con una vacuna. En los niveles doce y trece, durante el año 1912, el jugador examinará otros lugares geográficos de Cataluña. Finalizará su recorrido en el nivel catorce, durante el año 1809, donde tendrá que lidiar contra un grupo de radicales que han incendiado las fábricas de la ciudad. 


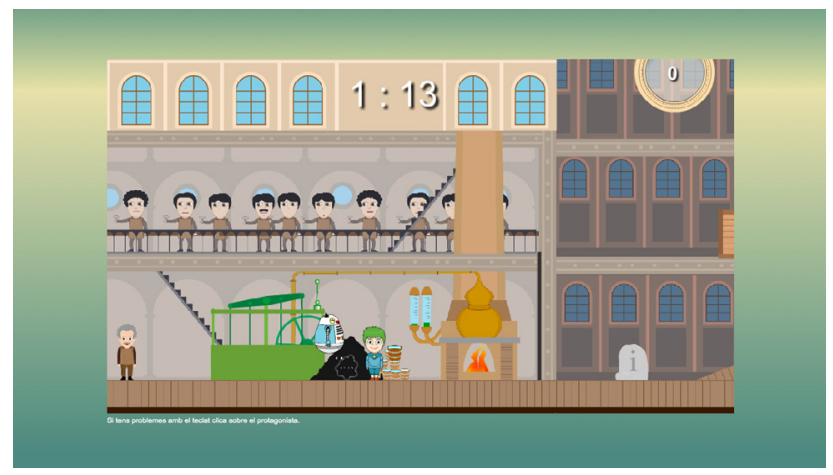

\section{Competencias}

Los contenidos forman parte de los objetivos, las reglas y los retos del juego, y la dinámica del mismo consiste en mostrar información estrechamente relacionada con las competencias del currículo del departamento de enseñanza de Cataluña. Esta combinación de conocimientos, capacidades y actitudes adecuadas, que se enmarca en un proyecto de desarrollo personal y colectivo, se aprovecha para trabajar diversos aspectos que pueden ampliarse en el aula.

\section{Las competencias básicas trabajadas son:}

1. Competencia comunicativa lingüística y audiovisual. Mediante la lectura, el jugador comprende información histórica y geográfica variada para poder avanzar; paralelamente debe interpretar contextos visuales.

2. Competencia cultural. El hecho de que se trabajen aspectos históricos ayuda a que el alumnado conozca, comprenda y valore críticamente las diferentes manifestaciones culturales e históricas.

\section{Competencias metodológicas:}

1. Competencia digital. Se potencia el aprendizaje de habilidades y estrategias en tecnologías digitales.

2. Competencia de aprender a aprender. El juego facilita que el alumnado aprenda por sí mismo habilidades técnicas y le proporciona instrumentos para que investigue y profundice en el conocimiento. 


\section{Competencias personales:}

1. El desarrollo del juego facilita que cada alumno supere de manera autónoma los conocimientos a medida que lea y supere niveles.

Otros conceptos, actitudes y procedimientos considerados específicos del ciclo superior de primaria y que se trabajan en el desarrollo de las actividades son:

- Conocer el medio social y entender el contexto histórico vivido por el personaje.

- Situarse en el espacio, orientándose y desplazándose en mapas. Utilizar espacios virtuales.

- Conocer los aspectos más relevantes de la vida en el siglo XX, la revolución industrial, los conflictos y avances tecnológicos.

- Situar etapas y hechos de la historia catalana en el tiempo y con una estructura cronológica.

- En el área de matemáticas, reconocer fracciones propias como parte de una unidad. Esto se enmarca en la disciplina de cálculo y proporciones.

- En el área de las lenguas, practicar la conversación, la comprensión de textos y mejorar la capacidad crítica para comprender información recibida. Además, se fomenta el conocimiento de otras lenguas mediante un vocabulario básico.

La actividad se puede plantear para que el alumno juegue en casa de forma individual o en grupo y que después en el aula ponga en común las experiencias como punto de partida de los temas de aprendizaje que propone el juego. Por otra parte, existe la posibilidad de utilizar el juego en la escuela, en grupo, según la disponibilidad de los ordenadores con el fin de ampliar información.

\section{VALORACIÓN DE LA EXPERIENCIA}

Son necesarios más principios y propuestas para la investigación y el diseño de experimentos y metodologías basadas en el diseño de juegos. Por tanto, esta propuesta, basada en la metodología investigación-acción, puede llegar a ser una solución práctica para el diseño de un serious game. La experiencia llevada a cabo por un grupo multidisciplinario ha permitido comprobar que es posible aplicar un proceso de cocreación y utilizar la investigación-acción como metodología para el diseño de un serious game que tiene como objetivo desarrollar competencias de aprendizaje. La metodología IA permite mejorar el conocimiento del proceso de cocreación necesario para el diseño de un juego de estas características. Al plantearse diversos principios, la conclusión obtenida es una clara comprensión de los juegos, así como del funcionamiento de la tecnología que debe utilizarse. Además, el marco 
teórico se orienta al diseño y proporciona una valiosa comprensión de cuestiones generales. Los informes de cada ciclo proporcionan diagnósticos útiles para todos los participantes y permiten identificar principios de diseño. Lo más relevante y destacable del uso de la IA es que el desarrollo de un prototipo se materializa teniendo en cuenta los resultados de los grupos de discusión, los resultados de la revisión bibliográfica y el análisis de los productos existentes en el mercado. El desarrollo del documento de diseño, el diseño del prototipo y el estudio de usabilidad permiten resumir y reunir los resultados posteriormente. Paralelamente, gracias al uso de esta metodología, ha sido posible explorar las necesidades de los alumnos, examinar oportunidades, concretar las competencias y la temática del juego, así como sus componentes estructurales: objetivos, reglas, retos e interacción. Todo ello ha dado como resultado un juego digital que permite trabajar competencias de educación orientadas a estudiantes de primaria.

Sintetizar los resultados y los conocimientos adquiridos a partir de un estudio de investigación-acción que involucra diferentes estrategias e intervenciones de recolección de datos permite generar un conjunto de principios de diseño que pueden aplicarse a un sistema de cocreación (Eguia-Gómez, Contreras-Espinosa y Albajes, 2014). Estos principios pueden contribuir a la literatura existente, que nos recomienda utilizar la cocreación en algunos momentos (Dahan y Hauser, 2002; Von Hippel, 2005; Kangas, 2010; Nambisan y Nambisan, 2008; Nambisan y Baron, 2009; Mouws y Bleumers, 2015), pero además ayudan a generar sistemas para la cocreación virtual (Kohler, Fueller, Matzler y Stieger, 2001) y apoyar a la literatura centrada en el aprendizaje basado en juegos digitales, porque, como mencionan Arnab et al. (2015), y Perrotta et al. (2013), todavía necesitamos más principios y propuestas para la investigación y el diseño de experimentos y metodologías que permitan trabajar en el diseño de juegos educativos. Asimismo, es necesario mirar hacia el uso de métodos mixtos como una opción que puede desplegarse en diferentes etapas, incluyendo desde la recogida de datos hasta el análisis de los mismos (Mayer et al., 2013; Lieberoth y Roepstorff, 2015).

Consideramos que esta experiencia contribuye a dar nueva información a los marcos de diseño existentes y proporciona a otros desarrolladores de juegos un sistema de cocreación flexible, dinámico y fácil de replicar. Realizar un proceso en el cual los profesores, como usuarios, cuenten con un papel activo, les ofrece la oportunidad de participar en un proceso de innovación y a los desarrolladores nos da la posibilidad de crear "obras" que los usuarios considerarán propias.

Por otro lado, al utilizar la IA no sólo se crean las bases para un sistema (Lindgren, Henfridsson, y Schultze, 2004), también se ponen de manifiesto las necesidades reales de los usuarios. Si la experiencia satisface a las necesidades hedónicas de los participantes, los esfuerzos realizados habrán valido la pena porque, como usuarios finales, serán colectivamente capaces de resolver problemas que antes no podrían haber enfrentado o superado (Von Ahn, 2008). Son ellos, los profesores, los usuarios responsables de que los juegos lleguen a las aulas. 
Somos críticos y consideramos que aún se necesita más investigación para mejorar la metodología utilizada, o incluso para comparar otros métodos utilizados en la cocreación de juegos, con el fin de arrojar mayores resultados sobre las cuestiones "cuándo" y "cómo" es mejor emplearlos y con qué tecnología. Vemos la necesidad de generar futuras investigaciones centradas en la eficacia de generar principios de diseño basados exclusivamente en la cocreación. En cuanto al desarrollo del juego, las limitaciones con las que nos hemos encontrado han sido básicamente limitaciones tecnológicas. Nos referimos a las mismas que puede encontrar cualquier equipo de desarrollo al contar con un presupuesto limitado y sin el apoyo de una gran productora. Por supuesto, el prototipo generado en esta experiencia no puede ser comparable tecnológicamente a los proyectos de grandes empresas de videojuegos. Pero con los recursos limitados se ha conseguido un producto que cumple con las expectativas del grupo de investigación y que podrá ponerse a prueba en las aulas.

\section{CONCLUSIONES}

En este proyecto se propuso una experiencia de cocreación con profesores de primaria que tuvieron un papel activo en el diseño de un serious game. Los objetivos planteados en el proyecto pudieron cumplirse: 1) Desarrollar un proceso de cocreación como este, en el que los usuarios contaran con un papel activo, ofreció a los profesores la oportunidad de participar en un proceso de innovación que resultó positivo. Se consideraron usuarios por ser los responsables de que los juegos lleguen a las aulas. 2) El proceso permitió además seleccionar un tema específico y las competencias del currículo del departamento de enseñanza de Cataluña que se introdujeron en el juego. Estas se trabajaron en relación con las necesidades pedagógicas específicas del ciclo superior de primaria. Las competencias constituyen un "saber hacer" complejo y adaptativo que se aplica de forma reflexiva y es susceptible de adecuarse a una diversidad de contextos, por consiguiente, todos los participantes de esta experiencia han considerado positivo el uso de dichas competencias en un juego digital. Además de introducirlas, se tuvo en cuenta que el juego debería contar con una jugabilidad atractiva para los alumnos. La idea principal, en este sentido, es que no perciban el juego como una actividad aburrida. Una jugabilidad atractiva permite medir la calidad de la experiencia de un jugador al interactuar con él en pruebas futuras y esto permitiría obtener nuevos indicadores. 3) La experiencia de cocreación permitió también definir una dinámica de trabajo durante todo el proceso, que puede repetirse fácilmente en otros proyectos y contextos. Pero, como se ha demostrado, para configurar adecuadamente un proceso de cocreación es necesario contar con metodologías estandarizadas.

Resaltamos que el trabajo en un equipo multidisciplinar es enriquecedor y necesario para este tipo de proyectos, pero plantea la necesidad de conjugar diferentes puntos de vista, objetivos y prioridades en su desarrollo. La comunicación constante es un punto básico y fundamental para realizar un trabajo multidisciplinar 
de estas características donde cada profesional aporta su conocimiento y experiencia en la consecución de unos objetivos comunes. Debe ponerse incluso mayor énfasis en la dimensión de la sociabilidad, descrita en los principios diseñados por Nambisan y Nambisan (2008), en el caso de los usuarios. El prototipo desarrollado, como resultado de la experiencia, nos ha ayudado además a:

a. Definir el perfil de los profesores usuarios y sus características. Ésto ha sido posible gracias a los grupos de discusión.

b. Elegir las competencias necesarias para un juego específico. Éste fue un elemento básico que se adaptó a las necesidades pedagógicas del currículo de primaria.

c. Garantizar la interoperabilidad del juego teniendo en cuenta los dispositivos utilizados habitualmente en las aulas catalanas, entre los que se encuentran los ordenadores y, en algunos casos, los dispositivos móviles.

Como trabajo futuro, planeamos estructurar nuevas sesiones de cocreación para refinar el método propuesto en este artículo. Es necesario contar con un diseño riguroso buscando validar su efectividad realizando nuevas experiencias. También nos proponemos diseñar un sistema con un enfoque de investigación-acción que permita diseñar y evaluar juegos, pero con la ayuda de alumnos de primaria, que, como usuarios finales, puedan participar del sistema de cocreación.

\section{Agradecimientos}

Agradecemos al Comissionat per a Universitats $i$ Recerca de la Generalitat de Catalunya por la financiación otorgada para la realización del juego Ferran Alsina.

\section{REFERENCIAS BIBLIOGRÁFICAS}

Abt, C. (1970). Serious Games. New York: Viking Press

Arnab, S., Lim, T., Carvalho, M., Bellotti, F., De Freitas, S., Louchart, S., Suttie, N., Berta, R., y De Gloria, A. (2015). Mapping learning and game mechanics for serious games analysis. British Journal of Educational Technology, 46(2), 391-411.

Banks, J., y Humphreys, S. (2008). The Labour of User Co-Creators Emergent Social Network Markets? Convergence, 14(4), 401-418. doi: http://dx.doi. org/10.1177/1354856508094660
Banks, J., y Potts, J. (2010). Co-creating games: a co-evolutionary analysis. New Media \& Society 12(2), 253-270. doi: 10.1177/1461444809343563

Barnett, R. (2001). Los límites de la competencia. El conocimiento, la educación superior y la sociedad. Barcelona: Gedisa.

Eguia-Gómez, J. L., Contreras-Espinosa, R. S., y Albajes, L. (2014). Co-creation of a serious game to develop comptences on the primary education program. Information Systems and Technologies 
(CISTI), $20149^{\text {th }}$ Iberian Conference, (pp. 1-6). doi:10.1109/CISTI.2014.6877080

Dahan, E., y Hauser, J. (2002). The Virtual Customer. Journal of Product Innovation Management, 19(5), 332-353. doi: http:// dx.doi.org/10.1111/1540-5885.1950332

DeSeCo (2005). The definition and selection of key competencies, resumen ejecutivo, OCDE.

Durugbo, C., y Pawar, K. (2014). A unified model of the co-creation process. Expert Systems with Applications: An International Journal, 41(9), 43734.387. doi: http://dx.doi.org/10.1016/j. eswa.2014.01.007

Gee, J. P. (2007). What video games have to teach us about learning and literacy. New York: Palgrave Macmillan.

Gibson, D., Aldrich, C., y Prensky, M. (2007). Games and simulations in online learning: Research and development frameworks. Hershey: Information Science Publishing.

Hine, G. S. (2013). The importance of action research in teacher education programs. Issues in Educational Research, 23(2), 151-163.

Holter, A. C., y Frabutt, J. M. (2012). Mission driven and data informed leadership. Catholic Education: A Journal of Inquiry and Practice, 15(2), 253-269.

Ibáñez Moreno, A., Jordano de la Torre, M., y Vermeulen, A. (2016). Diseño y evaluación de VISP, una aplicación móvil para la práctica de la competencia oral. RIED. Revista Iberoamericana de Educación a Distancia, 16(1), 63-81. doi: http://dx.doi. org/10.5944/ried.19.1.14580

Johnson, W. L. (2010). Serious Use of a Serious Game for Language Learning. International Journal of Artificial Intelligence in Education, 2O, 175-195.

Kangas, M. (2010). Creative and playfull learning: Learning through game cocreation and games in a playful learning environment. Thinking Skills and
Creauivitu, 5(1), 1-15. doi: http://dx.doi. org/10.1016/j.tsc.2009.11.001

Kebritchi, M., Hirumi, A., y Bai, H. (2010). The effects of modern mathematics computer games on mathematics achievement and class motivation, Computers \& Education, 55, 427-443.

Kemmis, S., y McTaggart, R. (1988). The action research planner. Geelong, Australia: Deakin University Press.

Kohler, T., Fueller, J., Stieger, D., y Matzler, K. (2011). Avatar-based innovation: consequences of the virtual co-creation experience. Computers in Human Behavior, 27(1), 160-168. doi: http:// dx.doi.org/10.1016/j.chb.2010.07.019

Kohler, T., Fueller, J., Matzler, K., y Stieger, D. (2001). Co.creation in Virtual Worlds: The design of the User Experience. MIS QUarterly, 35(3), 773-778.

Lieberoth, A., y Roepstorff, A. (2015). Mixed methods in games research, playing on strengths and countering weaknesses. En P. Lankoski y B. Staffan (Coords.), Game research methods: An overview (271289). Pittsburgh, USA: ETC Press.

Lindgren, R., Henfridsson, O., y Schultze, U. (2004). Design Principles for Competence Management Systems: A Synthesis of an Action Research Study. MIS Quarterly, 28(3), 435-472.

Marton, F. (1981). Phenomenography: Describing Conceptions of the World Around Us. Instructional Science, 10(2), 177-200. doi: http://dx.doi.org/10.1007 BFo0132516

Mayer, I., Bekebrebe, G., Wamelink, H., y Zhou, Q. (2013). A Brief Methodology for Researching and Evaluating Serious Games and Game-Based Learning. En T. Conolly, T. Hainey, E. Boyle, G. Baxter y P. Moreno-Ger (Coords.), Psychology, Pedagogy, and Assessment in Serious Games (357-393). Hershey, USA: IGI Global. 
Michael, D. R., y Chen, S. (2006). Serious games: Games that educate, train, and inform. Mason: Paperback.

Mouws, K., y Bleumers, L. (2015). Co-creating Games with Children: A case Study. International Journal of Gaming and Computer-Mediated Simulations, 7(3), 22-43. doi: http://dx.doi.org/10.4018/ IJGCMS.2015070102

Nambisan, P., y Nambisan, S. (2008). How to Profit from a better 'Virtual Customer Environment'. MIT Sloan Management Review, 49(3), 53-61. doi: http://dx.doi. org/10.1225/SMR279

Nambisan, S., y Baron, R. A. (2009). Virtual Customer Environments: Testing a Model of Voluntary Participation in Value CoCreation Activities. Journal of Product Innovation Management, 26(4), 388406. doi: http://dx.doi.org/10.1111 j.1540-5885.2009.00667

Perrenoud, Ph. (2012). Cuando la escuela pretende preparar para la vida. ¿Desarrollar competencias o enseñar otros saberes? Barcelona: Graó.
Perrotta, C., Featherstone, G., Aston, H., y Houghton, E. (2013). Game-based Learning: Latest Evidence and Future Directions. NFER Research Programme: Innovation in Education. Recuperado de http://ocw.metu.edu.tr/pluginfile. $\mathrm{php} / 10919 / \mathrm{mod}$ resource/content/1/ GAME01.pdf

Sawyer, B.(2007). Serious games: Broadening Games Impact Beyond Entertainment. Computer Graphics Forum, 26(3). doi: 10.1111/j.1467-8659.2007.01044.x.

Von Ahn, L., Maurer, B., McMillen, C., Abraham, D., y Blum, M. (2008). reCAPTCHA: Human-Based Character Recognition via Web Security Measures. Science, 321, 1465-1468. Recuperado de http://www.sciencemag.org/ content $/ 321 / 5895 / 1465$

Von Hippel, E. (2005). Democratizing Innovation. Cambridge: The MIT Press.

Zyda, M. (2005). From visual simulation to virtual reality to games. Computer, 38(9), 25-32. doi:10.1109/MC.2005.297.

\section{PERFIL ACADÉMICO Y PROFESIONAL DE LOS AUTORES}

Ruth S. Contreras-Espinosa. Doctora en Ingeniería Multimedia. Profesora de la Facultad de Empresa y Comunicación y de la Facultad de Ciencias y Tecnología en la Universitat de Vic-Universitat Central de Catalunya. Miembro del grupo Research group on Data and Signal Processing de la misma Universidad.

E-mail: ruth.contreras@uvic.cat

\section{DIRECCIÓN DE LA AUTORA}

Facultad de Empresa y Comunicación

Universitat de Vic-Universitat Central de Catalunya

Despacho E001

C/Sagrada Familia 7, 08500, Vic 
José Luis Eguia-Gómez. Doctor en Comunicación Interactiva. Profesor en la Escuela Técnica Superior de Ingeniería de Barcelona en la Universitat Politècnica de Catalunya. Miembro del grupo GIE (Grup d'Informàtica a l'Enginyeria) de la misma Universidad.

E-mail: eguia@ege.upc.edu

\section{DIRECCIÓN DEL AUTOR}

Escuela Técnica Superior de Ingeniería de Barcelona

Universitat Politècnica de Catalunya

Planta 8

Av. Diagonal 647, 08028, Barcelona

Lluis Solano Albajes. Doctor Ingeniero Industrial. Profesor titular de Universidad en la Escuela Técnica Superior de Ingeniería de Barcelona en la Universitat Politècnica de Catalunya. Miembro del grupo GIE (Grup d'Informàtica a l'Enginyeria) de la misma Universidad.

E-mail: solano@lsi.upc.edu

\section{DIRECCIÓN DEL AUTOR}

Escuela Técnica Superior de Ingeniería de Barcelona

Universitat Politècnica de Catalunya

Planta 8, Despacho 69

Av. Diagonal 647, 08028, Barcelona

Fecha de recepción del artículo: 19/11/2015

Fecha de aceptación del artículo: 02/03/2016

\section{Como citar este artículo:}

Contreras-Espinosa, R. S., Eguia-Gómez, J. L., y Solano Albajes, L. (2016). Investigación-acción como metodología para el diseño de un serious game. RIED. Revista Iberoamericana de Educación a Distancia, 19(2), pp. 71-90. doi: http:// dx.doi.org/10.5944/ried.19.2.15624 